Wright State University

CORE Scholar

Physics Faculty Publications

Physics

$1-1-1990$

\title{
Theoretical Intensity-Dependent Response of Nonlinear Periodic Structures
}

\author{
Paul A. Gohman \\ Gust Bambakidis \\ Wright State University - Main Campus, gust.bambakidis@wright.edu \\ Robert J. Spry
}

Follow this and additional works at: https://corescholar.libraries.wright.edu/physics

Part of the Physics Commons

\section{Repository Citation}

Gohman, P. A., Bambakidis, G., \& Spry, R. J. (1990). Theoretical Intensity-Dependent Response of Nonlinear Periodic Structures. Journal of Applied Physics, 67 (1), 40-44.

https://corescholar.libraries.wright.edu/physics/115

This Article is brought to you for free and open access by the Physics at CORE Scholar. It has been accepted for inclusion in Physics Faculty Publications by an authorized administrator of CORE Scholar. For more information, please contact library-corescholar@wright.edu. 


\title{
Theoretical intensity-dependent response of nonlinear periodic structures
}

\author{
Paul A. Gohman and Gust Bambakidis \\ Physics Department, Wright State University, Dosyton, Ohio 45435 \\ Robert J. Spry ${ }^{\text {e) }}$ \\ Wright Research and Development Center, Wright-Patterson Air Force Base, Ohio 45433
}

(Received 24 March 1989; accepted for publication 18 September 1989)

\begin{abstract}
We have modeled the response of a nonlinear periodic structure by means of the Abeles $2 \times 2$ matrix method. Our structure differs from the usual rejection-band filter designs, in that we have chosen the filter elements to be index matched in the absence of radiation, providing a rejection band that both grows and shifts as a function of incident intensity. The intensity output function of the model not only directly demonstrates optical bistability, but also limiting, switching, self-pulsing, and chaos.
\end{abstract}

\section{RATRODUCTION}

Nonlinear periodic structures have recently received much theoretical attention, both because of the importance of such devices for optical communications and computing, and because of the intrinsically interesting results, such as bistability, ${ }^{1-6}$ limiting, ${ }^{5}$ chaos, ${ }^{2}$ and soliton behavior. ${ }^{4,6-9}$

The usual theoretical approaches start with the slowiy varying envelope approximation, in which the electric fields in the periodic structure are taken to be the product of a rapidly oscillating spatial function, and a slower varying envelope function. Solutions to Maxwell's wave equation are then obtained by numerical methods, ${ }^{4,6}$ or by coupled-mode theory, ${ }^{1}$ Floquet-Block theory, ${ }^{7,8}$ and other analytic methods. ${ }^{5,9}$

Following an alternative numerical approach, we have solved the onedimensional wave equation in a nonlinear periodic structure by means of the Abelés matrix method that is commonly used in linear optical filter design. ${ }^{10}$ We believe our method has the advantages of simplicity, general applicability, and displaying the major known nonlinear phenomena in a straightforward manner.

\section{H. METHODOLOGY}

\section{A. Parameters}

Our numerical model was chosen to be a one-dimensional approximation for a nonlinear version of the crystalline colloidal array filter presently being experimentally developed. ${ }^{11-13}$ In this filter, the colloidal spheres are arranged in a crystalline structure that Bragg difiracts visible light as atomic crystals Bragg diffract $x$-ray radiation. Approximating a lattice of polystyrene spheres suspended in a nonlinear medium, the filter model has alternating linear and nonlinear layers, each with 100 nm thickness (the diameter of a polystyrene colloidal sphere) and linear refractive index of 1.6 (polystyrene) in the absence of radiation. The specific filter we are modeling is one in which the alternating layers are index matched in the absence of radiation, producing only a fiat transmittance as a function of wavelength. As the input intensity increases, a filter notch simultaneously develops at a predetermined wavelength and also broadens in an unusual fashion.

\footnotetext{
"Author to whom correspondence should be addressed.
}

The theoretical justification for treating the diffraction of radiation passing through a three-dimensional crystal by the one-dimensional Abelés method was first stated by Berreman. ${ }^{14}$ This method may be used if the variation of the refractive index normal to any set of Bragg planes is known, the variation being obtained from the real part of the complex structure factor for these planes. Our intention here is not to extract the exact one-dimensional refractive index profile by the Fourier analysis procedure outined by Berreman, but rather to solve a closely related problem, that of a quarter-wave stack whose alternating refractive indices have the same values as those of the colloidal spheres and the host medium. Our mathematical technique has much wider applicability than that for the crystalline colloidal array fiter, for example, the nonlinear response of semiconductor-based interference filters, a subject of a great deal of recent research activity. ${ }^{15,16}$

The refractive index of the nonlinear layers varies with intensity within any layer $m$ as

$$
n_{m}=n_{0}+n_{2} I_{m}
$$

where $n_{0}$ is the linear refractive index and $n_{2}$ is the intensitydependent nonlinear coefficient. For simplicity and to avoid boundary effects, the refractive index of 1.6 was chosen for boundary media. The nonlinear coefficient $\left(n_{2}=6.56 \times 10^{-13} \mathrm{~cm}^{2} / \mathrm{W}\right.$ ) for the nonlinear layers represents a fast responding optical material such as MNA (2methyl-4-nitroanitine) ${ }^{17}$ In the absence of radiation, the linear refractive index of MNA is assumed to be matched to 1.6 by mixing with other organic materials having lower values of linear refractive index. With incident radiation, the index of the nonlinear layers increases and produces a feedback mechanism with a central rejection wavelength near $640 \mathrm{~nm}$. The results presented in this paper were derived from a 1000 -period structure $\left[(H L)^{1000} H\right]$ illustrated in Fig. 1 , where $H$ represents a high-index nonlinear layer and $L$ represents the low-index linear layer. To further simplify modeling the nonlinear filter, absorption was neglected and incident radiation was normail to the layers.

\section{Matrix method}

Assuming an isotropic medium and a linearly polarized field, the one-dimensional wave equation for our case is of 


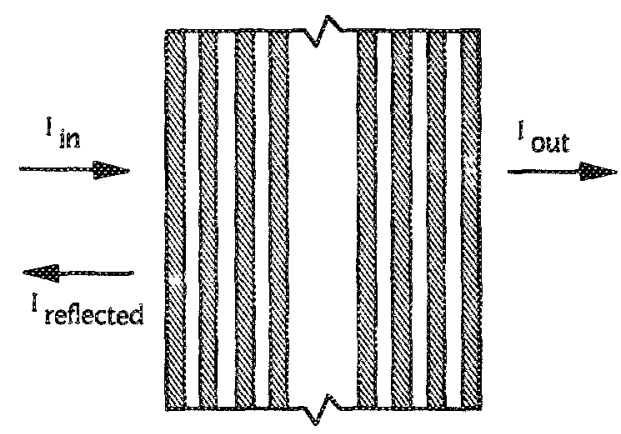

FIG. 1. Simple schematic of 1000 -period structure $\left[(H L)^{1000} H\right]$ with hatched layers representing nonlinear layers. All iayers are $100 \mathrm{~nm}$ thick.

the form

$$
\frac{\partial^{2} E(z, t)}{\partial z^{2}}=\frac{[n(z, E)]^{2}}{c^{2}} \frac{\partial^{2} E(z, t)}{\partial t^{2}}
$$

where $E(z, t)$ is the transverse component of the electric field and $n(z, E)$ is the spatially dependent and field-dependent refractive index. In order to determine the intensity-dependent refractive index of the nonlinear layers, it is necessary to calculate the electric field within the layered structure. To accomplish this, we treat each layer as a thin film and use the characteristic matrix approach as described by Heavens, ${ }^{10}$

$$
\left(\begin{array}{l}
E_{f, m} \\
E_{b, m}
\end{array}\right)=M_{m}\left(\begin{array}{l}
E_{f, m+1} \\
E_{b, m+1}
\end{array}\right)
$$

Here $E_{f, m}$ and $E_{b, m}$ are the forward and backward traveling electric fields, respectively, in the $m$ th layer. Because the backward field at the output ( $E_{b, \text { sut }}$ ) equals zero, it is possible to propagate the forward field $\left(E_{f, \text { out }}\right)$ backwards from the output medium towards the incident medium using successive applications of the $2 \times 2$ transformation matrix

$$
M_{m}=\frac{1}{t_{m+1}}\left(\begin{array}{cc}
\exp \left(i \delta_{m}\right) & r_{m+1} \exp \left(i \delta_{m}\right) \\
r_{m+1} \exp \left(-i \delta_{m}\right) & \exp \left(-i \delta_{m}\right)
\end{array}\right),
$$

where $t_{m+1}$ and $r_{m+1}$ represent the Fresnel coefficients at the boundary that separates layers $m$ and $m+1$, $\delta_{m}=2 \pi \delta_{m} d_{m} / \lambda$ is the optical phase thickness, and $d_{m}$ is the physical thickness of layer $m$. Starting with a unity output field ( $\left.E_{\text {out }}=\left|E_{f, \text { out }}\right|=1\right)$, the relative fields in each layer and the incident fiejd $\left(E_{\text {in }}=\left|E_{f, \text { in }}\right|\right)$ are determined, and the field amplitude in each layer is normalized with respect to $E_{\mathrm{i} x}$ by

$$
\left|E_{f, m}\right|=\frac{\left|E_{f, m}\right|}{E_{\mathrm{in}}}, \quad\left|E_{b, m}\right|=\frac{\left|E_{b, m}\right|}{E_{\mathrm{in}}} .
$$

The intensities in each layer are then calculated with

$$
I_{m}=\left|E_{m}\right|^{2} E_{\text {in }} / 4
$$

where

$$
E_{m}=E_{f, m}+E_{b, m}
$$

Prior to Eq. (6a), our calculations assumed the electric flelds were static in determining the intensity within the structure. By taking a temporal average of the two coupled waves, a correction factor of $1 / 2$ is included in Eq. (6a) to account for dynamic wave propagation. Also, the temporally averaged intensity function is not constant throughout a layer. To simplify and speed up calculations, an averaging technique is used to provide a single intensity to drive the noninear response of each layer; consequently, an additional factor of $1 / 2$ is included in Eq. (6a) to account for spatial averaging.

Transmission spectra are produced by holding the nonlinear indices constant and calculating

$$
T=\frac{I_{\text {out }}}{l_{\text {in }}}=\frac{n_{\text {out }}\left(E_{\text {our }}\right)^{2}}{n_{\text {in }}\left(E_{\text {in }}\right)^{2}}
$$

as a function of wavelength. Thus, this method provides a self-consistent numerical solution to the noninear problem, in which there are no simplifying approximations other than averaging the spatial intensity [Eq. $(6 a)]$ prior to updating the index [Eq. (1)] of each nonlinear layer. The effect of this averaging was shown to be small by more extensive calculations in which the layers were extensively segmented, and the results from the two different methods compared.

\section{Algorithm}

Since the nonlinear refractive indices are a function of input intensity $\left(I_{\text {in }}\right)$, a method was devised to determine the output intensity $\left(I_{\text {out }}\right)$ given an input function. By defining the leading edge of a light pulse to have a gradually increasing intensity, rather than an abrupt step intensicy, our input function starts at zero and increases via small discrete steps to a maximum intensity $\left(I_{\max }\right)$. With this technique, the interdependent nonlinear indices and electric fields change slowly as the input increases in a ramping manner. The following algorithm defines the process in more detail.

(i) $I_{\text {in }}=0$.

(ii) Calculate normalized electric fields, Eqs. (3) to (5).

(iii) Calculate output intensity, Eq. (7).

(iv) Update nonlinear indices, Eqs. (1) and (6).

(v) Increment input intensity, $I_{\text {in }}=I_{\text {in }}+\Delta I$.

(vi) If $I_{\mathrm{in}}<I_{\mathrm{max}}$, then go to step (ii) or else stop.

Other input functions are required to model the output response of the filter, such as the triangle function for bistability and steady state for self-pulsing and chaos. The triangle function emulates the leading and trailing edge of a pulse that produces the hysteresis loop of a bistable output. The algorithm is modified to represent a triangle pulse by decreasing $I_{\text {in }}$ after reaching $I_{\max }$. The steady-state input involves ramping to $I_{\max }$ and then holding $I_{\mathrm{in}}=I_{\max } \mathrm{con}$ stant. We assume an instantaneous nonlinear material response time and associate each iteration with one transir time $\left(n_{0} L / c\right)$, where $L$ is the physical thickness of the structure.

A numerical matrix method has also been developed by Bovard and Macleod to study nonlinear narrow-band rejection filters having originally unmatched layers. ${ }^{8}$ Their method consists of using a step input and iterating until the nonlinear indices reach steady state. Although they also obtain bistable behavior, our method and our demonstration of other phenomena are substantially different from their results. 


\section{RESULTS AND DISCUSSION}

\section{A. Limiting}

Starting with index-matched layers, an initially transparent filter will acquire a rejection band cue to the intensity-induced mismatch between the linear and nonlinear layers. With a positive nonlinear susceptibility, the total refractive index of the nonlinear layers increases with intensity and changes the Bragg condition to a longer wavelength. Consequently, the rejection band grows and shifts to longer wavelengths as the input intensity is increased in a ramp function. If the rejection band shifts towards the wavelength of the incident radiation, a limiting output function will develop.

Figure 2 illustrates a limited output intensity followed by a region of instability. The dashed line represents a transparent response $\left(I_{\text {out }}=I_{\text {in }}\right)$. Transmission spectra were calculated at three input intensities (points $A, B$, and $C$ ) to show the growth and shift of the rejection band (Fig. 3 ). The vertical line in Fig. 3 represents the radiation wavelength $(640.5 \mathrm{~nm})$ that is at the long-wavelength side of the rejection band at low intensity (point $A$, Fig. 3). As the input intensity increases, the rejection-band peak grows and shifts towards the radiation wavelength (points $B$ and $C, F i g .3$ ). Within the structure, the electric field envelope can be described as an exponential function with a more rapid decay at higher input intensities.

Once the input intensity reaches a certain threshold, the exponential envelope breaks down, allowing more radiation. into the structure. This will increase the nonlinear refractive indices and the optical path length of the filter whereby the rejection band shifts beyond the radiation wavelength, which will be somewhere in the side lobes on the short-wavelength side of the rejection band. With the radiation wavelength in the side lobes, the output intensity is unstable, as seen in Fig. 2 for $I_{\text {in }}$ greater than $40 \mathrm{GW} / \mathrm{cm}^{2}$.

\section{B. Bistability}

Using the same filter parameters as for the limiter, but at a different radiation wavelength $(640.05 \mathrm{~nm})$, a bistable out-

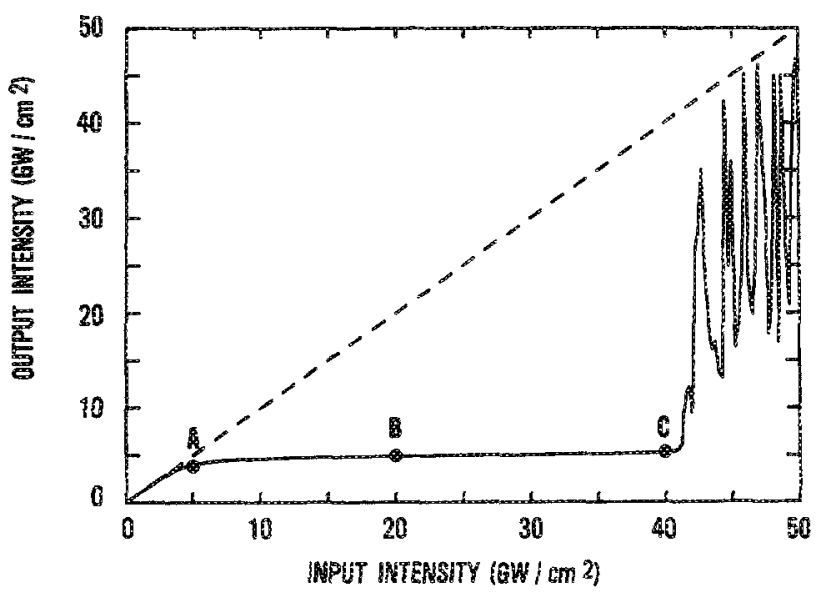

FIG. 2. Transmitted intensity as a function of input intensity at $640.5 \mathrm{~nm}$, illustrating a broad region of limiting tehavior ultimately followed by chaos.

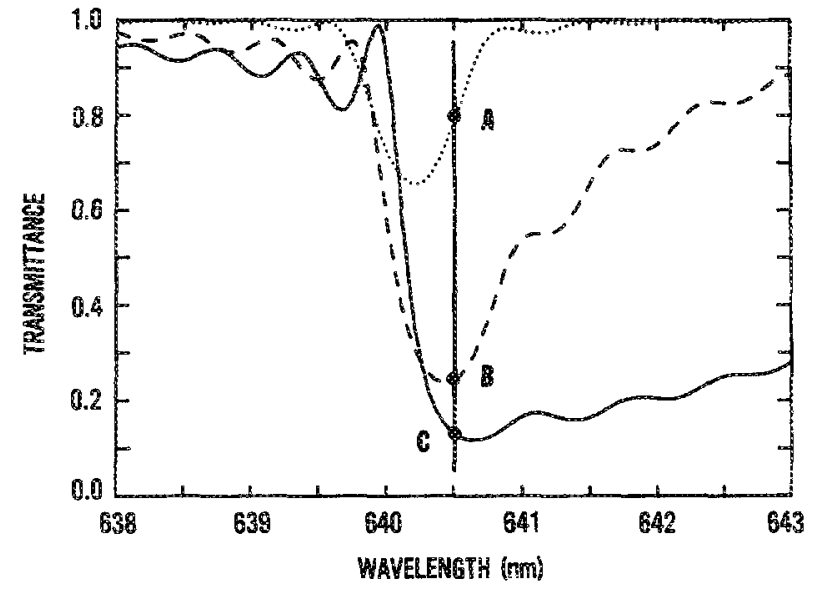

FIG. 3. Transmission spectra calculated at three input intensities of the limiting curve (Fig. 2): dotted curve, $5 \mathrm{GW} / \mathrm{cm}^{2}$; dashed curve, 20 $\mathrm{GW} / \mathrm{cm}^{2}$; solid curve, $40 \mathrm{GW} / \mathrm{cm}^{2}$.

put is obtained (Fig. 4). We used a triangle input intensity function where $I_{\text {in }}$ was ramped to $I_{\max }\left(20 \mathrm{GW} / \mathrm{cm}^{2}\right)$, then decreased to zero. With increasing input, the output intensity follows smoothly through points $A$ and $B$ and then jumps to $100 \%$ transmission at $I_{\text {in }}=12 \mathrm{GW} / \mathrm{cm}^{2}$. By increasing the input further, the temporal output oscillations become unstable at approximately $14 \mathrm{GW} / \mathrm{cm}^{2}$. This behavior will be discussed later under self-pulsing and chaos. With decreasing input, the output does not retrace its original path through point $B$, but rather, follows a path through point $C$. The ewo different output intensities at $I_{\text {in }}=10 \mathrm{GW} / \mathrm{cm}^{2} \mathrm{can}$ be understood in terms of the electric field envelopes. At point $B$, the envelope is described by an exponential function, whereas at point $C$, the envelope forms a "gap soliton" in the terminology of Chen and Mills. ${ }^{4,6}$ This field structure seems quite stable and requires less input intensity to maintain; consequently, as the input intensity decreases, $100 \%$ transmission is maintained. As the input intensity decreases further, the soliton dissipates and the output returns to the single-valued region. The resulting hysteresis loop is bistable.

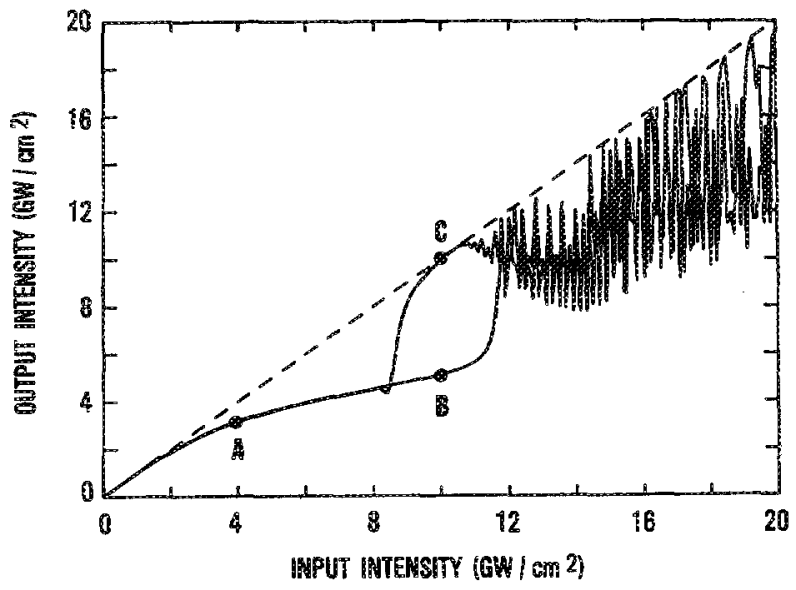

FIG. 4. Transmitted intensity as a function of input intensity at $640.05 \mathrm{~mm}$, illustrating regions of bistability, self-pulsing, and chaos. 


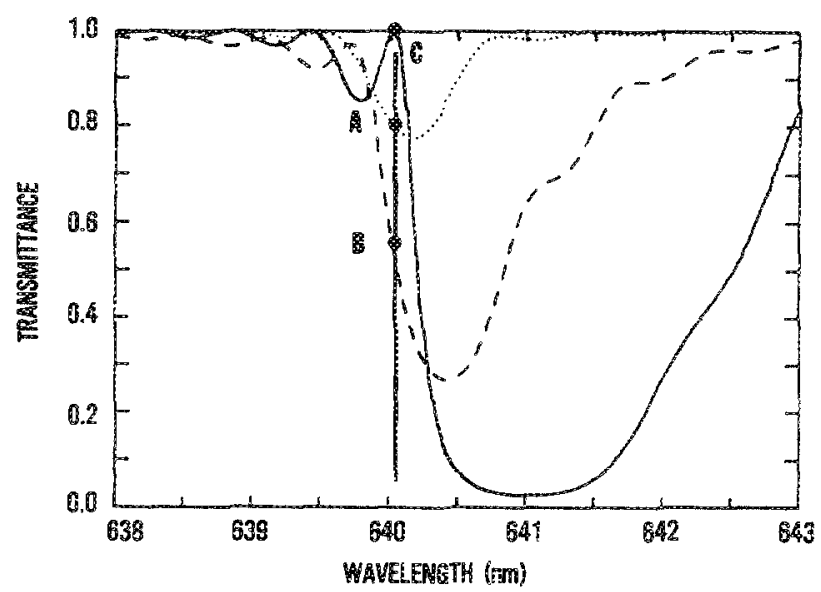

FIG. 5. Transmission spectra calculated at three input intensities of the bistability curve (Fig. 4): dotted curve, $4 \mathrm{GW} / \mathrm{cm}^{2}$; dashed curve, 10 $\mathrm{GW} / \mathrm{cm}^{2}$ (low state); solid curve, $10 \mathrm{GW} / \mathrm{cm}^{2}$ (high state).

The bistability can also be described with respect to the transmission spectra of the filter. At points $A, B$, and $C$ of Fig. 4 , the nonlinear indices were held constant while the transmission spectra in Fig. 5 were calculated. At low input intensity (point $A$ ), the radiation wavelength is on the shortwavelength side of the rejection band; as the input increases, the rejection band grows and shifts toward longer wavelength. At a critical input intensity, the rejection band will jump to a longer wavelength while the input wavelength becomes positioned between the main lobe and the first side lobe at point $C$. At this point, all the incident radiation is passing through the filter and tends to prevent the rejection band from shifting back when the input decreases.

In our bistability example, the switching intensity at 12 $\mathrm{GW} / \mathrm{cm}^{2}$ may appear inordinateiy large when power requirements are considered; however, the crystalline colloidal array fiter has the design fexibility that enables a bistable response at much lower input intensities. Lower switching intensities can be achieved with two different design changes: initially unmatched layer pairs and more layers. In the case of unmatched layers, a rejection-band already exists in the absence of incident radiation, which eliminates the need for the additional intensity to create the band. Additionally, increasing the number of periods results in a steeper slope on the side of the rejection band, thus requiring a smaller wavelength shift and consequently less intensity for a transition to the upper hysteresis state.

\section{Swltching}

Our studies have shown that the filter model will also demonstrate simple switching without bistability, provided that we slightly modify the filter design. If the nonlinear host media of the crystalline colloidal array filter has a lower linear index than the polystyrene spheres, incident radiation will increase the index of the nonlinear material to match the linear layers. This can be interpreted as transforming the grating from an index-unmatched to an index-matched condition, and the rejection band will shrink rather than grow as it shifts.

Figure 6 represents the output function, resulting from a

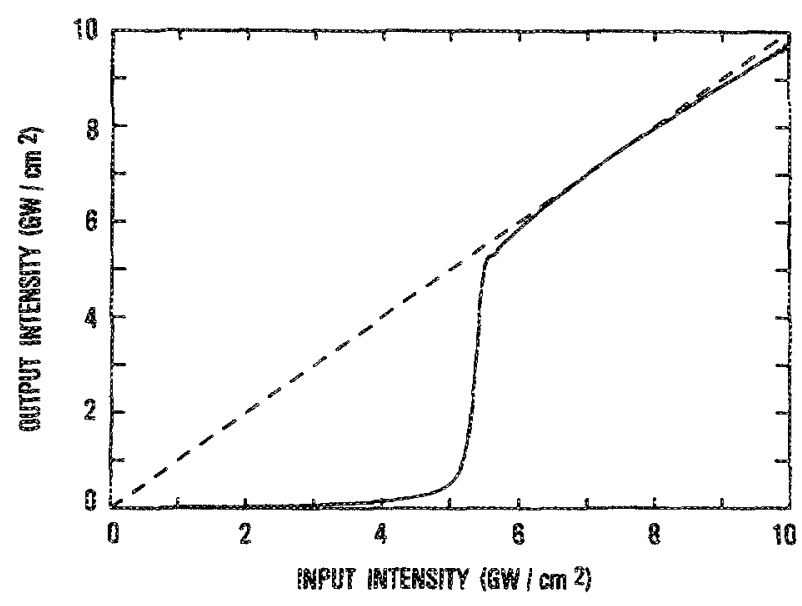

FIG. 6. Transmitied intensity as a function of input intensity at $640.0 \mathrm{~nm}$, illustrating simple, total switching.

ramp input function, of a 1000-period filter that has a linear index difference of 0.045 between the linear and nonlinear layers. The radiation wavelength $(640 \mathrm{~nm})$ is positioned within the rejection band at low intensities, and with increasing input intensity the rejection band shifts slightly until the band edge is encountered. A further intensity increase will cause an even more rapid shift of the band edge and allow even more light to enter the structure. When $100 \%$ transmission is reached, the rejection band, as well as side lobes, have diminished; consequently, lobe-induced oscillations are absent. With a quickly responding nonlinear material, the output will retrace its original path with decreasing input intensity.

\section{Selp-pukising and chaos}

Returning to the index-matched layers used for limiting and bistability, our model will demonstrate self-pulsing that becomes unstable with increasing input intensity. This phenomena occurs at two wavelength locations relative to the radiation-induced rejection band. Each case demonstrates different routes to chaos: period doubling and two-frequency, which are described by Harrison and Biswas. ${ }^{19}$ In both cases, the oscillations result from the rejection band beating against the radiation wavelength at a constant input intensity.

The first case occurs on the short-wavelength side of the rejection band and can be seen in the bistability plot (Fig. 4). Between 12 and $14 \mathrm{GW} / \mathrm{cm}^{2}$ input intensity, there are two overlapping oscillations corresponding to the ascending and descending sides of the triangle puise. Upon performing detailed calculations of this self-pulsing, we observe perioddoubling bifurcations as input intensity increases. Eventual$\mathrm{ly}$, the pulsing becomes unstable or chaotic above 14 $G W / \mathrm{cm}^{2}$ input intensity. An understanding of this pulsing can be gained by inspecting the transmission spectra (Fig. 5). The solid line spectrum oscillates about point $C$ with greater amplitude at higher input intensities. Interpreting the interlobe region as a potential well, the large spectrum oscillations will position the radiation wavelength in the nonlinear and asymmetric regions, producing a chaotic ternporal output. 

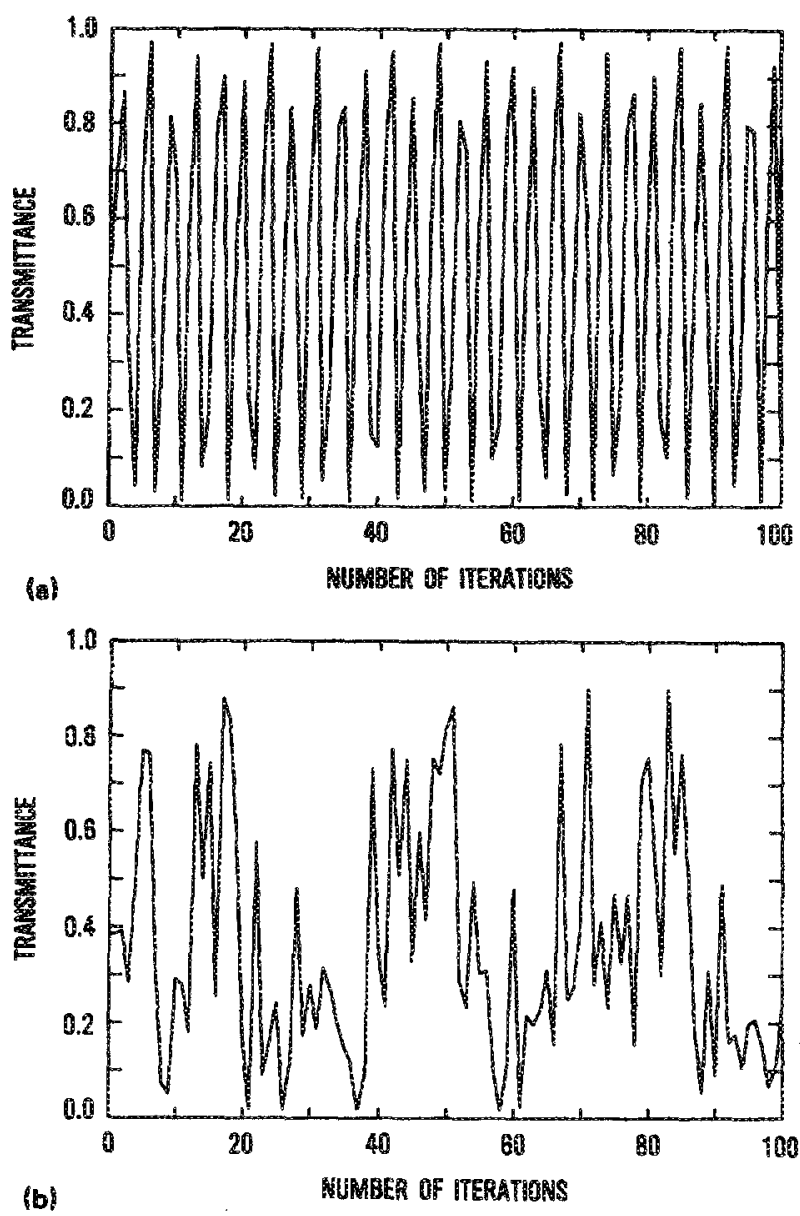

FIG.7. Output intensity at 641.0 nm with constant input intensity. (a) Selfpulsing at $I_{\mathrm{in}}=15 \mathrm{GW} / \mathrm{cm}^{2}$. (b) Chaos at $I_{\text {ico }}=90 \mathrm{GW} / \mathrm{cmi}^{2}$.

The second case occurs with radiation wavelengths on the long-wavelength side of the intensity-induced rejection band. Self-pulsing output [Fig. 7 (a)] occurs at $15 \mathrm{GW} / \mathrm{cm}^{2}$ and at a wavelength of $641 \mathrm{~nm}$, which is longer than that for the limiting example. The pulsing frequency will depend on the response time of the nonlinear medium as well as the thickness of the filter. Assuming an instantaneous response time, the pulsing frequency in Fig. 7(a) is calculated to be several hundred terahertz. Increasing the input intensity to $90 \mathrm{GW} / \mathrm{cm}^{2}$, the output will make a transition to chaos [Fig. $7(b)$ ] via the two-frequency route. A full discussion of the chaotic behavior of this filter is planned to be presented in a later paper.

\section{CONCLUSION}

The nonlinear matrix method has allowed us to model nonlinear periodic structures, such as the crystalline colloidal array filter, and has demonstrated a variety of optical functions essential for optical communications and computing. Hopefully, this model will facilitate the development of optical devices and an understanding of nonlinear phenomena.

\section{ACKNOWLEDGMENTS}

The authors wish to thank Charles L. Strecker, Richard L. Sutherland, Kent Kogler, and Everett Rea for many helpful discussions.

'H. G. Winful, J. H. Marburger, and E. Garmire, Appl. Phys. Lett. 35, 379 (1979).

${ }^{2}$ H. G. Winful and G. D. Cooperman, Appl. Phys. Lett. 40, 298 (1982).

${ }^{3}$ F. Delyon, Y.-E. Lévy, and B. Souillard, Plys. Rev. Lett. 57, 2010 (1986).

4. Chen and D. L. Mills, Phys. Rev. Lett. 58, 160 (1987).

A. Mecozzi, S. Trillo, and S. Wabnitz, Opt. Lett. 12, 1008 (198\%).

${ }^{6}$ W. Chen and D. L. Mills, Phys. Rev. B 36, 6269 (1987).

'J. E. Sipe and H. G. Winful, Opt. Lett. 13, 132 (1988).

${ }^{8}$ C. M. de Sterke and J. E. Sipe, Phys. Rev. A 38, 5149 (1988).

${ }^{9}$ D. L. Mills and S. E. Trullinger, Phys. Rev. B 36, 947 (1987)

${ }^{10}$ O. S. Heavens, Optical Properties of Thin Solid Films (Dover, New York, 1965).

"P. L. Flaugh, S. E. O'Donnell, and Sanford A. Asher, Appl. Spectrosc. 38, 847 (1984).

${ }^{12}$ R. J. Spry and D. J. Kosan, Appl. Spectrosc. 40, 782 (1986).

${ }^{13}$ S. A. Asher, P. L. Flaugh, and Guy Washinger, Spectroscopy 1, 26 (1986).

${ }^{14}$ D. W. Berreman, Phys. Rev. B 14, 4313 (1976)

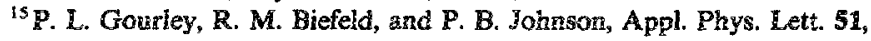
$1310(1987)$.

${ }^{16}$ O. Sahlén, J. Opt. Soc. Am. B 5, 82 (1988).

${ }^{77}$ T. Y. Chang, Opt. Eng. 20, 220 (1981).

${ }^{18}$ B. G. Bovard and H. Angus Macleod, J. Mod. Opt. 35, 1151 (1988).

${ }^{19}$ R. G. Harrison and D. J. Biswas, Nature 321, 394 (1986). 